Supplementary Information:

\title{
Influence of substitutional groups on the ordering and crystallization of amphiphilic silsesquioxanes at the air-water interface
}

\author{
Utsav $^{1}$, Wei Bu ${ }^{2}$, Binhua Lin ${ }^{2}$, Rupak Banerjee ${ }^{1 *}$ \\ ${ }^{1}$ Department of Physics, Indian Institute of Technology Gandhinagar, Palaj, Gandhinagar - 382355, Gujarat, India. \\ ${ }^{2}$ NSF's ChemMatCARS, University of Chicago, Chicago, IL 60637, USA. \\ *Email: rupakb@iitgn.ac.in
}


Figure S 1:
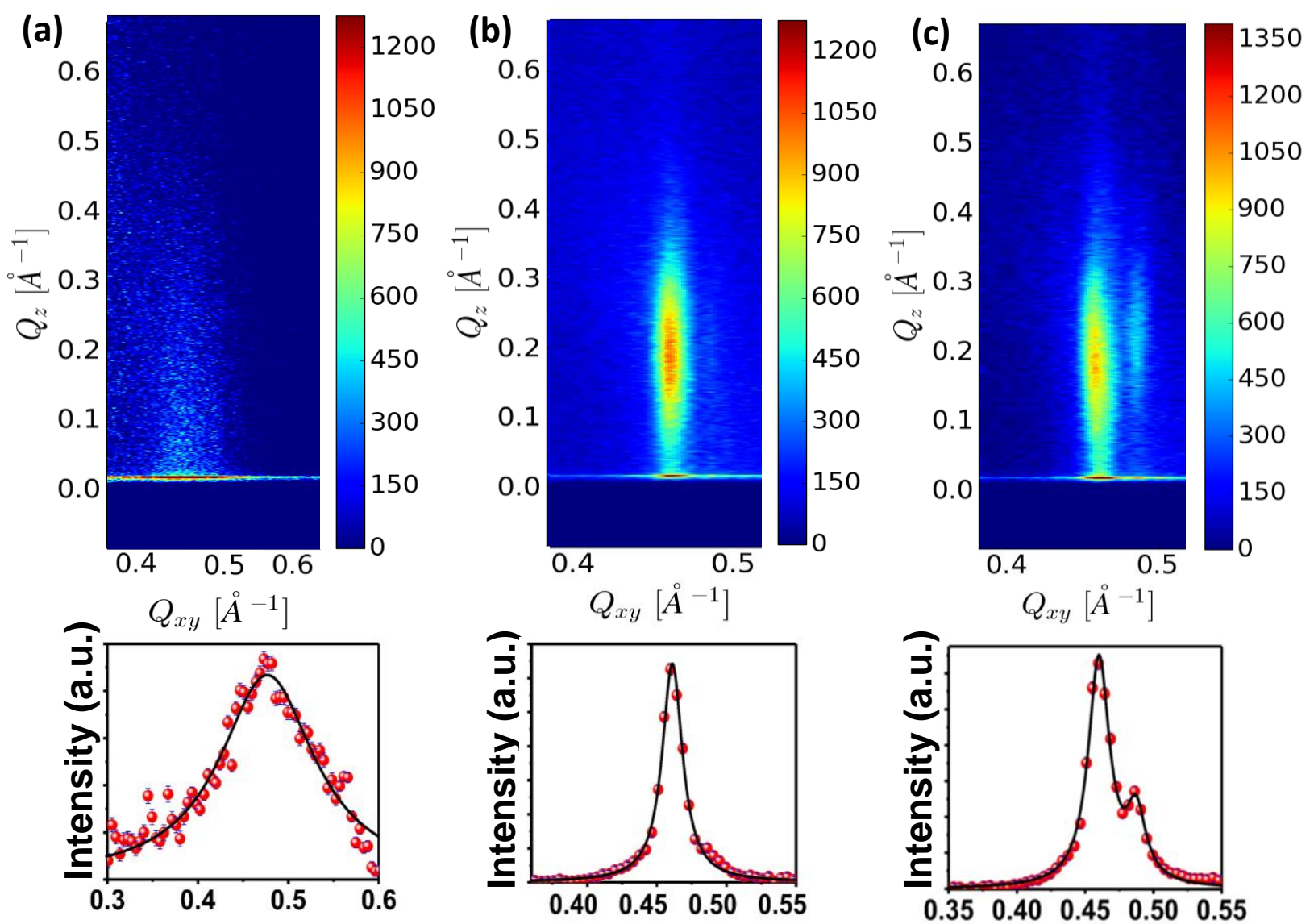

Figure S 1: GIXS diffractogram and corresponding integrated line-cut intensity profile observed over TCHPOSS layers at surface pressure (a) prior to collapse at $2.5 \mathrm{mN} / \mathrm{m}$ and weak correlation peak centered around $Q_{x y}=0.46 \AA^{-1}$, (b) at the collapse pressure $3.45 \mathrm{mN} / \mathrm{m}$, showing a single Bragg-rod diffraction profile centered around $Q_{x y}=0.46 \AA^{-1}$ and $Q_{z}=0.20 \AA^{-1}$, and (c) at extreme surface pressure of $20 \mathrm{mN} / \mathrm{m}$, showing twin Bragg-rod profile adding another peak centered around $Q_{x y}=0.48 \AA^{-1}$ and $Q_{z}=0.21 \AA^{-1}$, originating due to scattering from tilted dimes present within the bilayers. 
Figure S 2:
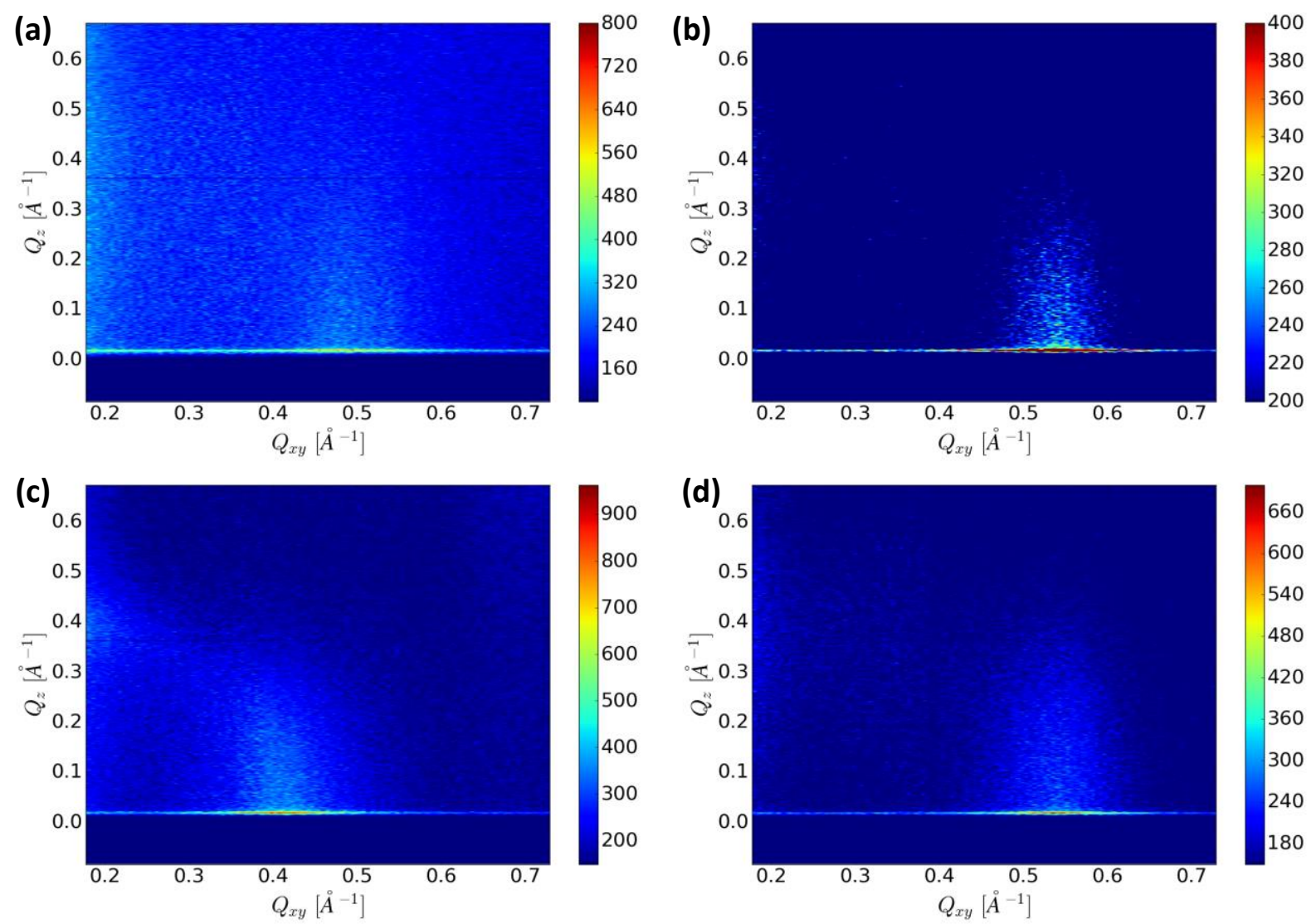

Figure S 2: GIXS measurement showing weak correlation peaks for decompressed layers of (a) TBPOSS, (b) TCHPOSS, (c) TOPOSS, and (d) DOBPOSS layers, originating from dimers decrystallized into the monomeric state. 
Figure S 3:

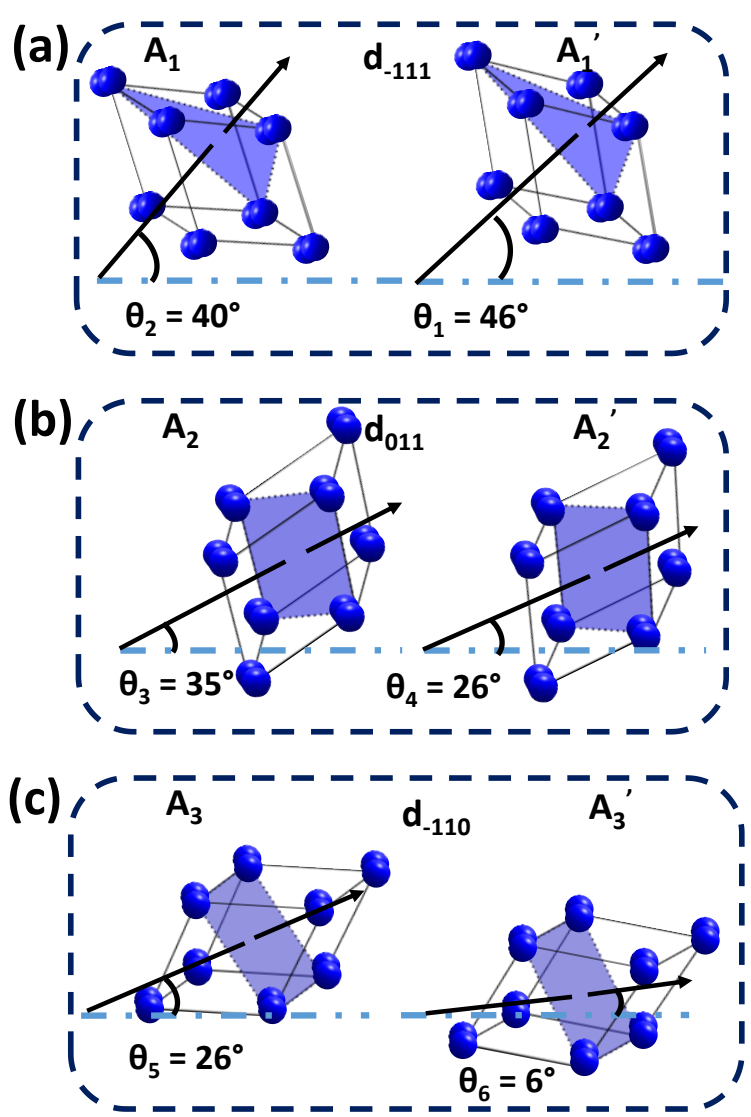

Figure S 3: Schematic representation of preferred tilt angles denoted by $\left(A_{1}, A_{2}\right.$, and $\left.A_{3}\right)$, subtended by crystal planes of TBPOSS dimers with respect to the interface for lattice planes of miller indices (a) -111 , (b) 011 , and (c) -110 , respectively.
Figure S 4:

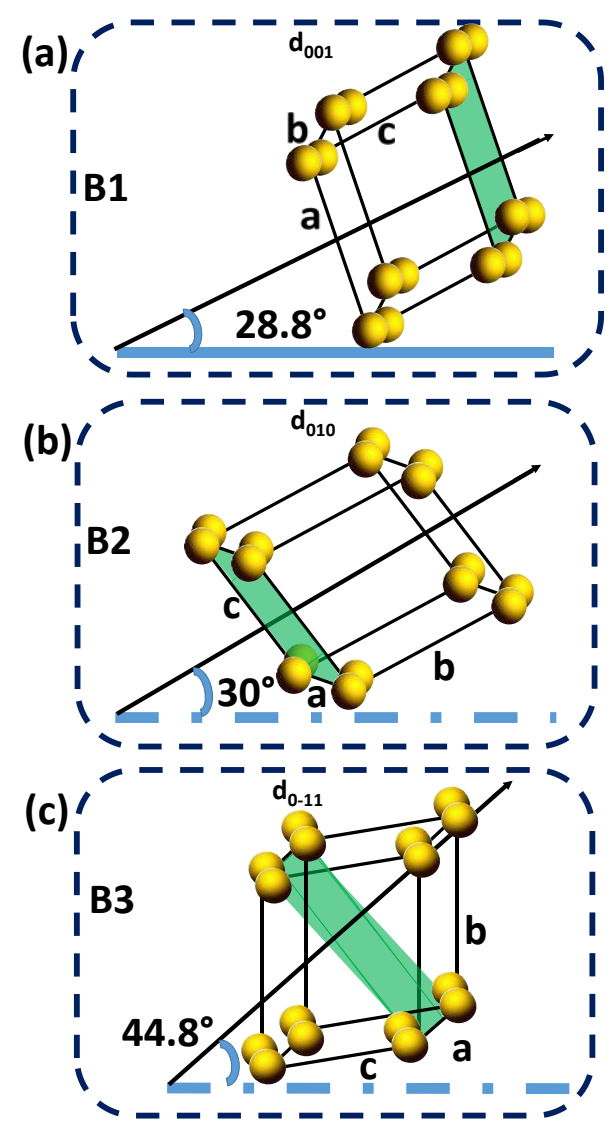

Figure S 4: Schematic representation of preferred tilt angles denoted by (B1, B2, and B3), subtended by crystal planes of TCHPOSS dimers with respect to the interface for lattice planes of miller indices (a) 001, (b) 010, and (c) 0-11, respectively. 
Figure S 5:
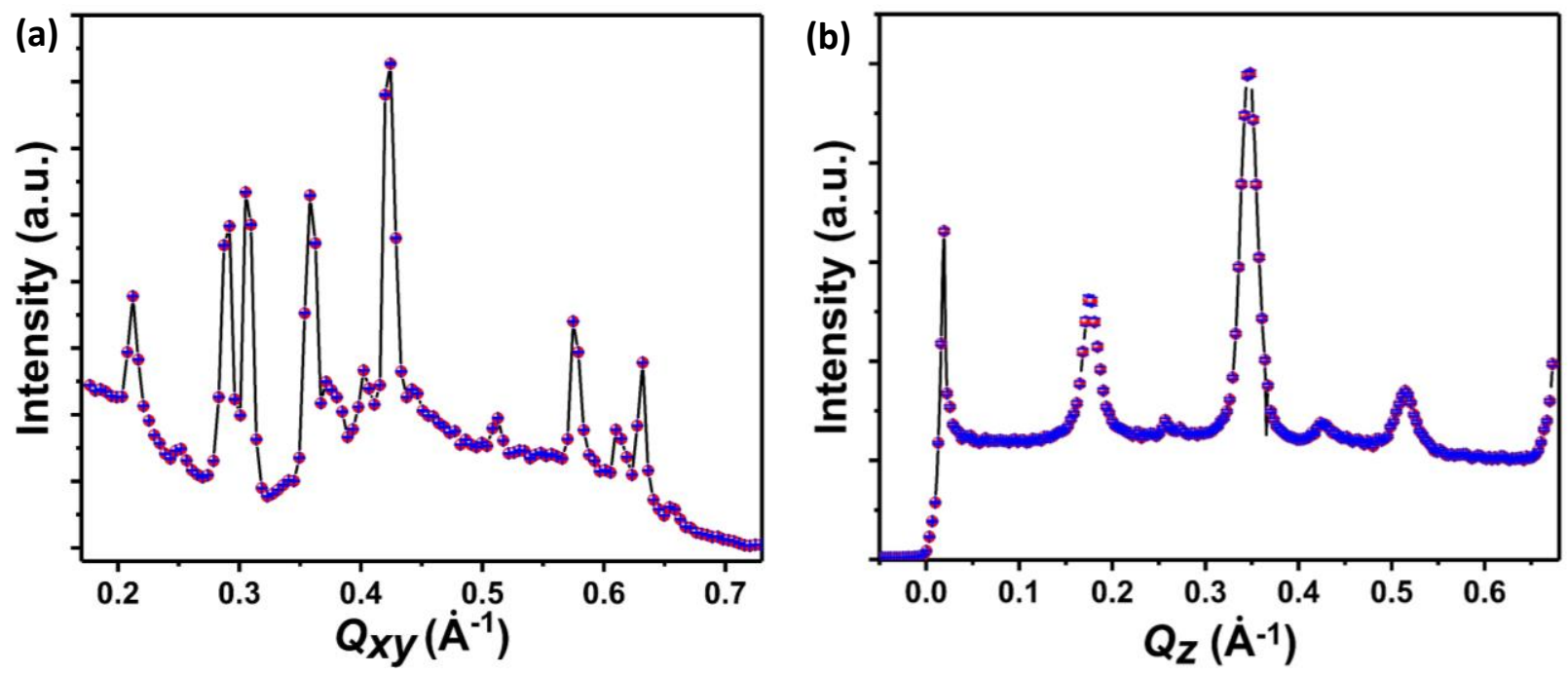

Figure S 5: Integrated intensity profile from GIXS measurement along (a) in-plane direction by summing $Q_{z}$ values from $0.0 \AA^{-1}$ to $0.63 \AA^{-1}$, and (b) out-of-plane direction by summing $Q_{x y}$ values from $0.2 \AA^{-1}$ to $0.72 \AA^{-1}$, performed over DOBPOSS layer at extreme compression of $20 \mathrm{mN} / \mathrm{m}$ during $2^{\text {nd }}$ compression cycle.

Figure S 6:
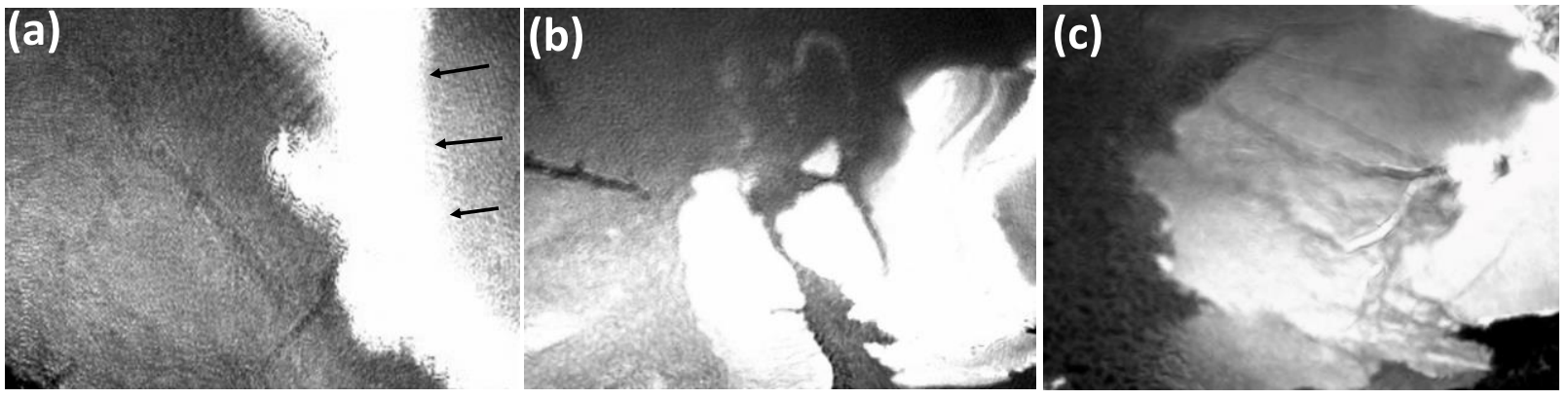

Figure S 6: BAM images of TCHPOSS layers at different surface pressure and area per molecule values for the $1^{\text {st }}$ compressiondecompression rate of $1 \mathrm{~mm} / \mathrm{min}$ at a $25^{\circ} \mathrm{C}$ temperature. The surface pressure values for imaging are (a) $3.45 \mathrm{mN} / \mathrm{m}$ showing monolayer to bilayer phase boundary marked by arrows, (b) $10 \mathrm{mN} / \mathrm{m}$ exhibiting a large proportion of dimeric domains at the interface during $1^{\text {st }}$ compression cycle, and (c) $2.5 \mathrm{mN} / \mathrm{m}$ during $1^{\text {st }}$ decompression, showing coexisting phases of monolayer and bilayer. 
Figure S 7:

(a)
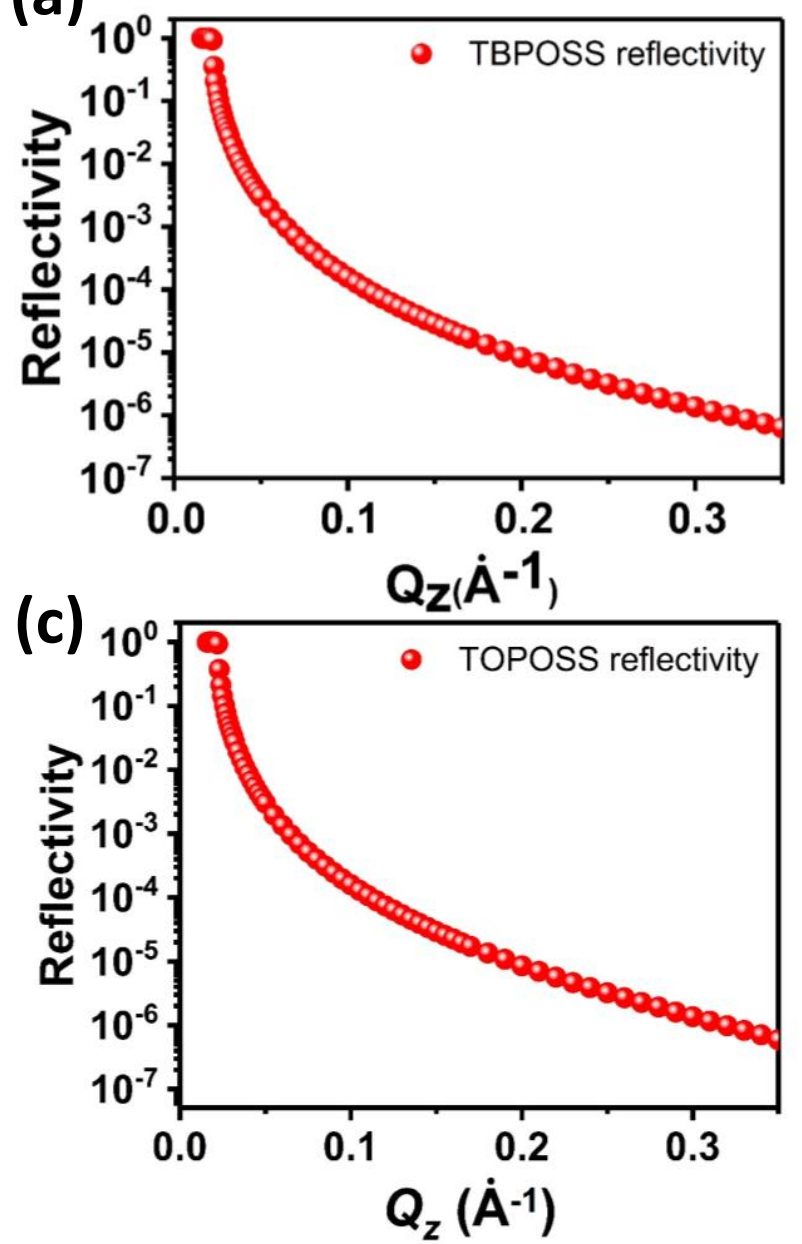

(b)
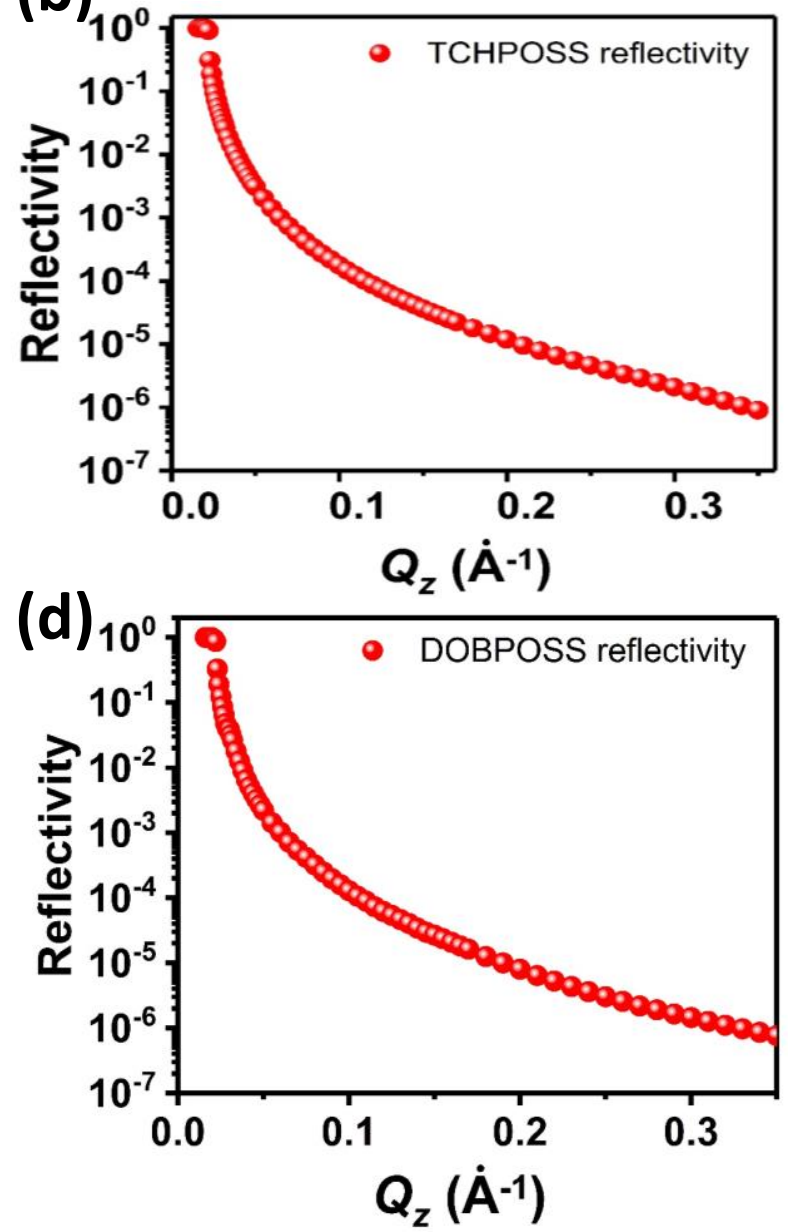

Figure S 7: X-ray reflectivity profiles measured over compressed floating layers of (a) TBPOSS, (b) TCHPOSS, (c) TOPOSS, and (d) DOBPOSS, respectively. The lack of electron density contrast between the sub-phase and the POSS layers prohibits any modulation in the reflectivity profile that can be analyzed to extract the out-of-plane ordering of the layers. 
Figure S 8:

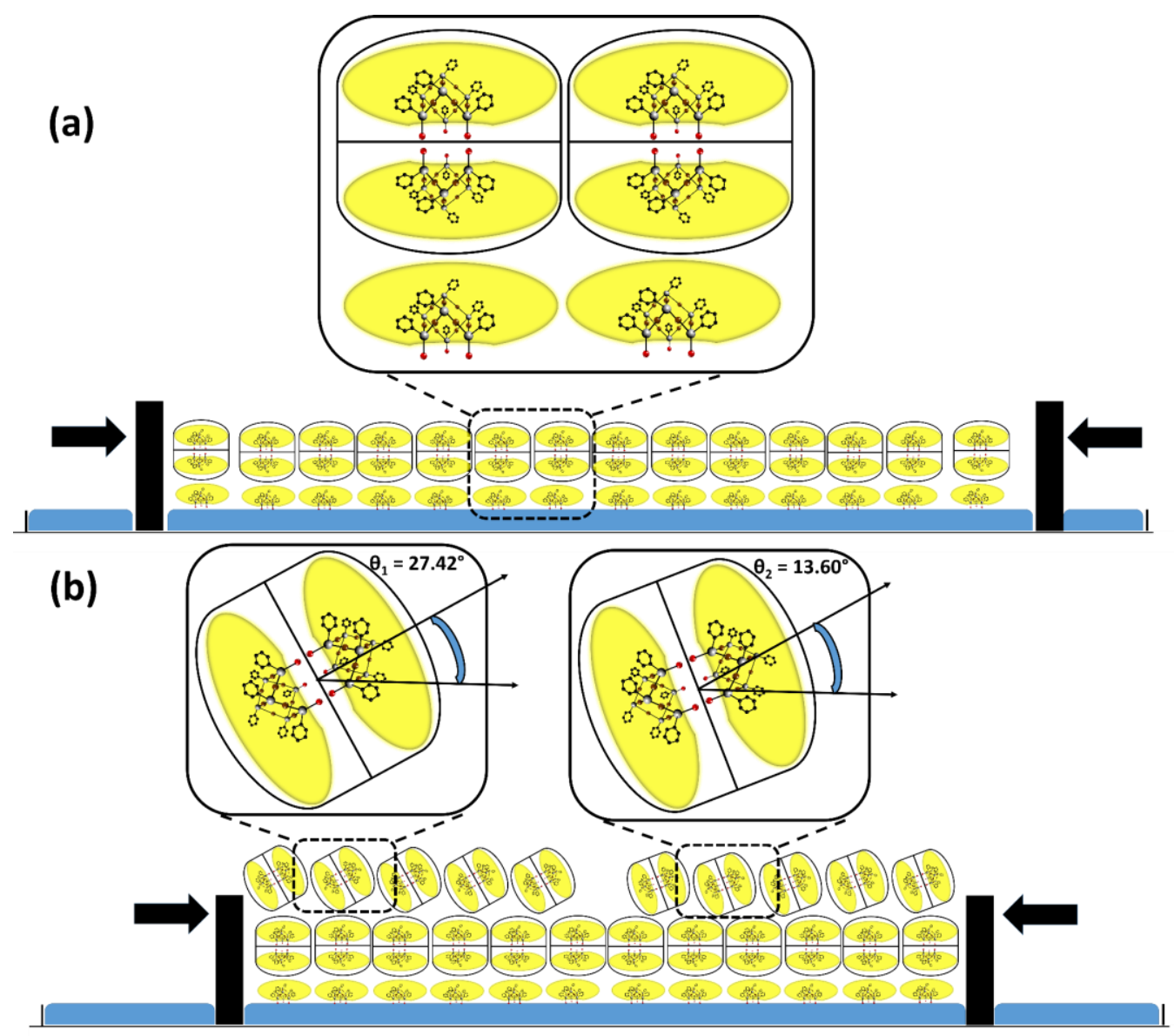

Figure S 8: Schematic illustration of the organization during (a) the $1^{\text {st }}$ compression cycle post-collapse at a surface pressure value of $3.45 \mathrm{mN} / \mathrm{m}$, showing the formation of an ordered layer of dimerized TCHPOSS, and (b) the $2^{\text {nd }}$ compression at a surface pressure of $15 \mathrm{mN} / \mathrm{m}$, showing bilayer formation of dimerized TCHPOSS with two different tilt angles in the top layer.

\section{Explanation of Bilayer formation:}

Compression of the interface leads to the monolayer's collapse wherein the monomers are squeezed out to form bilayers of dimerized TCHPOSS, as beyond the collapse pressure, the monomers transferred to the top of the monolayer undergo dimerization. A schematic rendering of the packing behavior of TCHPOSS molecules (monomers as well as bilayers formed out of dimers) within and above the monolayer is depicted in Figure S 8 (a). The monolayer ordering at the interface is manifested as the in-plane correlation peak around $Q_{x y}=0.5 \AA^{-1}$ in the GIXS profile. In contrast, the ordering in the dimer layer results in an intense Bragg rod diffraction profile. Lateral spacing between the dimers is more than that of the monolayer due to the comparatively stronger steric repulsion between the hydrophobic substituents of the TCHPOSS dimers.

Decompression of the TCHPOSS layer relaxes the interface, disrupting the above ordering throughout the interfacial area. The structural ordering post-collapse pressure during the second compression constitutes the bilayer assembly showing two tilt angles of $27.42^{\circ}$ (designated as $\theta_{1}$ ) and $13.60^{\circ}$ (designated as $\theta_{2}$ ), corresponding to the differences in the $Q_{z}$ values of the Bragg rod split along the $Q_{z}$ direction, but maintaining the same in-plane correlation length since there is no splitting of the Bragg rod along the $Q_{x y}$ direction. The corresponding schematic structure is shown in Figure $\mathrm{S} 8$ (b). 


\section{Figure S 9:}
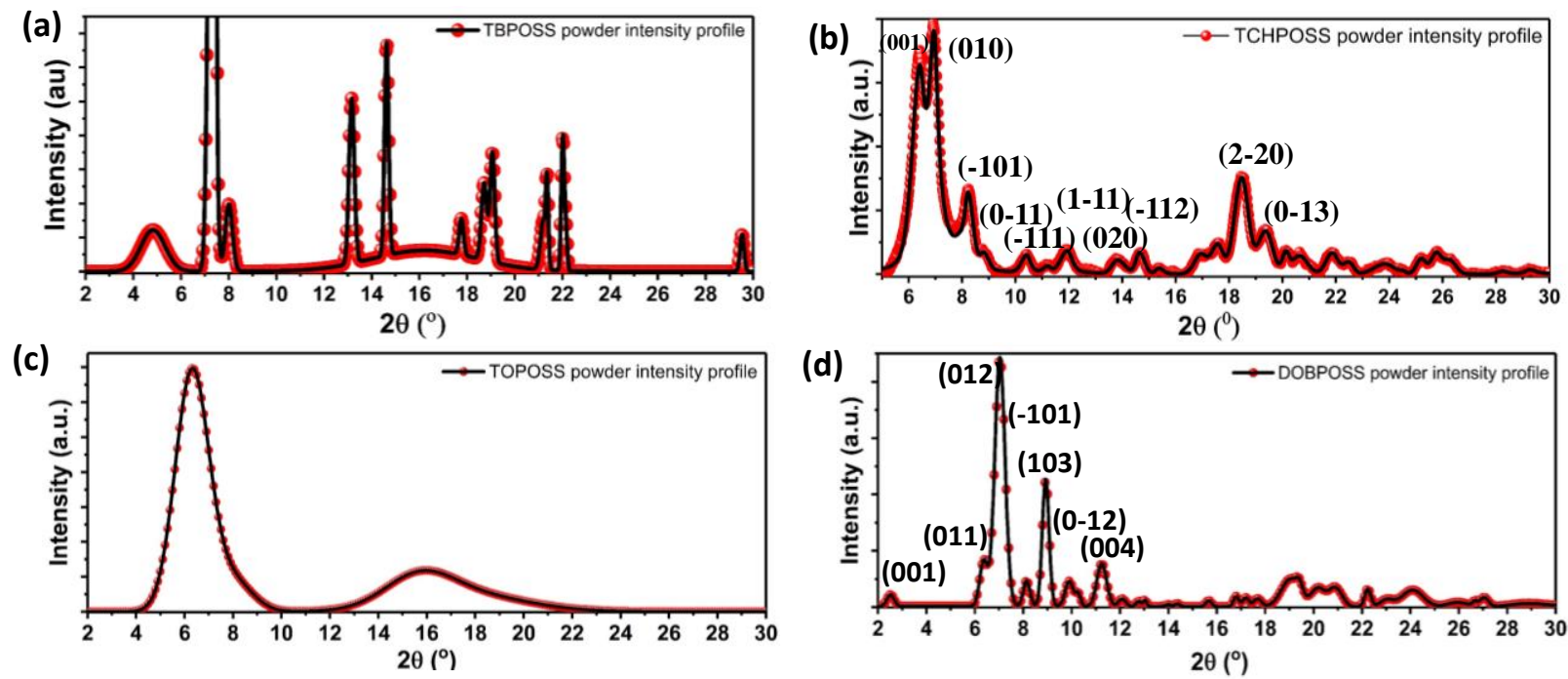

Figure S 9: X-ray diffractogram under $1.54 \AA \mathrm{Cu}-\mathrm{K}_{\alpha}$ illumination of powdered bulk POSS variants for (a) TBPOSS, (b) TCHPOSS, (c) TOPOSS, and (d) DOBPOSS, respectively. Miller indices corresponding to the prominent peaks of TCHPOSS and DOBPOSS are shown alongside. 


\section{Table S 1:}

Table S 1: List of observed and calculated values corresponding to different $\mathrm{d}_{\mathrm{hkl}}$ planes for TCHPOSS crystal formed at the air-water interface. The lattice planes are indexed with calculated lattice constants having triclinic $(\mathrm{P} \overline{1})$ lattice.

\begin{tabular}{|c|c|c|}
\hline Observed $d_{h k l}$ & Calculated $d_{\mathrm{hkl}}$ & Miller indices \\
\hline 13.920 & 13.919 & 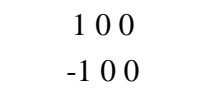 \\
\hline 12.762 & 12.719 & 001 \\
\hline 11.671 & 11.680 & $\begin{array}{ccc}0 & 1 & 0 \\
0 & -1 & 0\end{array}$ \\
\hline--- & 11.396 & $\begin{array}{ccc}1 & -1 & 0 \\
-1 & 1 & 0\end{array}$ \\
\hline --- & 10.767 & -101 \\
\hline 9.875 & 9.877 & $0-11$ \\
\hline --- & 8.659 & $1-11$ \\
\hline --- & 8.434 & 101 \\
\hline 8.312 & 8.325 & -111 \\
\hline --- & 7.721 & 011 \\
\hline --- & 7.607 & $\begin{array}{ccc}-1 & -1 & 0 \\
1 & 10\end{array}$ \\
\hline 7.528 & 7.562 & $-1-11$ \\
\hline 6.933 & 6.959 & $\begin{array}{rll}2 & 0 & 0 \\
-2 & 0 & 0\end{array}$ \\
\hline
\end{tabular}

Table S 2:

Table S 2: List of observed and calculated values corresponding to different $\mathrm{d}_{\mathrm{hkl}}$ planes for DOBPOSS crystals formed at the air-water interface. The lattice planes are indexed with calculated lattice constants having triclinic $(\mathrm{P} \overline{1})$ lattice.

\begin{tabular}{|c|c|c|}
\hline Observed $d_{\text {hkl }}$ & Calculated $d_{\text {hkl }}$ & Miller indices \\
\hline 15.963 & 15.963 & 201 \\
\hline 13.944 & 13.944 & -201 \\
\hline 13.649 & 13.649 & $2-21$ \\
\hline 12.729 & 12.729 & $0-22$ \\
\hline 12.633 & 12.632 & 112 \\
\hline 12.617 & 12.617 & 121 \\
\hline 12.070 & 12.069 & -112 \\
\hline 11.504 & 11.506 & $3-11$ \\
\hline 11.115 & 11.110 & -220 \\
\hline 10.632 & 10.632 & $1-13$ \\
\hline 10.174 & 10.174 & $-2-12$ \\
\hline--- & 10.190 & 310 \\
\hline 9.982 & 9.981 & $1-23$ \\
\hline 9.950 & 9.951 & 311 \\
\hline 9.836 & 9.835 & $3-22$ \\
\hline 9.459 & 9.459 & -103 \\
\hline 8.664 & 8.974 & 222 \\
\hline 8.890 & 8.890 & 312 \\
\hline 8.866 & 8.866 & -113 \\
\hline 8.644 & 8.645 & $3-13$ \\
\hline 8.128 & 8.127 & $-2-13$ \\
\hline 7.822 & 7.822 & $1-24$ \\
\hline 7.605 & 7.605 & 313 \\
\hline
\end{tabular}




\section{Table S 3:}

Table S 3: Calculated lattice parameters for TCHPOSS and DOBPOSS samples in powdered bulk form.

\begin{tabular}{|c|c|c|}
\hline Lattice & TCHPOSS & DOBPOSS \\
parameters & powder & powder \\
\hline $\mathrm{a}(\AA)$ & 12.55 & 14.69 \\
\hline $\mathrm{b}(\AA)$ & 13.07 & 14.81 \\
\hline $\mathrm{c}(\AA)$ & 14.66 & 35.66 \\
\hline$\alpha\left(^{\circ}\right)$ & 94.64 & 67.70 \\
\hline$\beta\left(^{\circ}\right)$ & 106.70 & 67.89 \\
\hline$\gamma\left({ }^{\circ}\right)$ & 98.94 & 71.13 \\
\hline
\end{tabular}

